\title{
Clinical validation of the 2017 international consensus guidelines on intraductal papillary mucinous neoplasm of the pancreas
}

\author{
Jae Seung Kang ${ }^{1, *}$, Taesung Park2,*, Youngmin Han', Seungyeon Lee ${ }^{3}$, Heeju Lim³, Hyeongseok Kim', \\ Se Hyung Kim ${ }^{4}$, Wooil Kwon ${ }^{1}$, Sun-Whe Kim¹, Jin-Young Jang ${ }^{1}$ \\ ${ }^{1}$ Department of Surgery and Cancer Research Institute, Seoul National University College of Medicine, Seoul, Korea \\ ${ }^{2}$ Department of Statistics and Interdisciplinary Program in Biostatistics, Seoul National University, Seoul, Korea \\ ${ }^{3}$ Department of Mathematics and Statistics, Sejong University, Sejong, Korea \\ ${ }^{4}$ Department of Radiology, Seoul National University College of Medicine, Seoul, Korea
}

\begin{abstract}
Purpose: The 2017 international consensus guidelines (ICG) for intraductal papillary mucinous neoplasm (IPMN) of the pancreas were recently released. Important changes included the addition of worrisome features such as elevated serum CA 19-9 and rapid cyst growth (>5 mm over 2 years). We aimed to clinically validate the 2017 ICG and compare the diagnostic performance between the 2017 and 2012 ICG.

Methods: This was a retrospective cohort study. During January 2000-January 2017, patients who underwent complete surgical resection and had pathologic confirmation of branch-duct or mixed-type IPMN were included. To evaluate diagnostic performance, the areas under the receiver operating curves (AUCs) were evaluated.

Results: A total of 448 patients were included. The presence of mural nodule (hazard ratio [HR], 9.12; 95\% confidence interval [Cl], 4.60-18.09; $\mathrm{P}=0.001)$, main pancreatic duct dilatation ( $>5 \mathrm{~mm}$ ) (HR, 5.32; 95\% Cl, 2.67-10.60; $\mathrm{P}=0.001)$, thickened cystic wall (HR, 3.40; $95 \% \mathrm{Cl}, 1.51-7.63 ; \mathrm{P}=0.003)$, and elevated CA 19-9 level ( $>37 \mathrm{unit} / \mathrm{mL})(\mathrm{HR}, 5.25 ; 95 \% \mathrm{Cl}$, 2.05-13.42; $\mathrm{P}=0.001$ ) were significantly associated with malignant IPMN. Malignant lesions showed a cyst growth rate $>5$ $\mathrm{mm}$ over 2 years more frequently than benign lesions $(60.9 \%$ vs. 29.7\%, $\mathrm{P}=0.012$ ). The AUC was higher for the 2017 ICG than the 2012 ICG (0.784 vs. 0.746).

Conclusion: The new 2017 ICG for IPMN is clinically valid, with a superior diagnostic performance to the 2012 ICG. The inclusion of elevated serum CA 19-9 level and cyst growth rate to the 2017 ICG is appropriate.

[Ann Surg Treat Res 2019;97(2):58-64]
\end{abstract}

Key Words: Guideline, Pancreatic ductal carcinoma, Pancreatic mucinous neoplasms

\section{INTRODUCTION}

In 1996, intraductal papillary mucinous neoplasm (IPMN) of the pancreas was designated as an independent disease by the World Health Organization (WHO) [1]. Whereas main- duct type IPMN has a high malignant potential and warrants surgical resection, surgical indications for branch-duct type IPMN (BD-IPMN) or mixed-type IPMN remain controversial due to their low malignant potential. The first and second editions of the international consensus guidelines (ICG) for the
Received May 2, 2019, Reviewed May 3, 2019,

Accepted June 26, 2019

Corresponding Author: Jin-Young Jang

Department of Surgery and Cancer Research Institute, Seoul National University College of Medicine, 101 Daehak-ro, Jongno-gu, Seoul 03080, Korea

Tel: +82-2-2072-2194, Fax: +82-2-741-2194

E-mail: jangjy4@snu.ac.kr

ORCID code: https://orcid.org/0000-0003-3312-0503
*Jae Seung Kang and Taesung Park contributed equally to this study as co-first authors.

Copyright (c) 2019, the Korean Surgical Society

(c) Annals of Surgical Treatment and Research is an Open Access Journal. All articles are distributed under the terms of the Creative Commons Attribution NonCommercial License (http://creativecommons.org/licenses/by-nc/4.0/) which permits unrestricted non-commercial use, distribution, and reproduction in any medium, provided the original work is properly cited. 
management of IPMN of the pancreas were introduced in 2006 and 2012, respectively [2,3]. The 2012 ICG proposed three highrisk stigmata (obstructive jaundice in a patient with a cystic lesion of the head of the pancreas, enhancing mural nodule $\geq 5 \mathrm{~mm}$, main pancreatic duct [MPD] size $\geq 10 \mathrm{~mm}$ ) and five worrisome features (cyst size $\geq 3 \mathrm{~cm}$, enhancing mural nodule $<5 \mathrm{~mm}$, thickened/enhancing cyst walls, 5-9 mm of MPD size, and abrupt change in caliber of the pancreatic duct with distal pancreatic atrophy). Patients who were diagnosed with BDIPMN under these guidelines were recommended to undergo complete surgical resection. One study reported that the 2012 ICG provided the practical validity for predicting malignant BDIPMN [4].

In 2015, more conservative guidelines were released by the American Gastroenterological Association (AGA) [5]. They suggested less aggressive resection criteria and more conservative surveillance criteria than the 2012 guidelines. However, some studies questioned the limited evidence of these guidelines and proposed their revision [6-8].

Recently, the new 2017 ICG were released [9]. Only limited changes have been introduced compared to the 2012 ICG, with the addition of two worrisome features, such as increased serum CA $19-9$ and a rapid cyst growth ( $>5 \mathrm{~mm}$ per 2 years) [10-12]. However, these features have not undergone rigorous clinical validation. Therefore, this study sought to investigate the usefulness of the parameters proposed by the 2017 ICG, clinically validate the 2017 ICG and compare the diagnostic performance between the 2017 and 2012 ICG.

\section{METHODS}

\section{Patients and materials}

This was a retrospective cohort study with prospectively collected clinicopathological data from electronic medical records in accordance with the protocol of our institution. We included patients admitted to the Department of Surgery of our hospital between January 2000 and January 2017 who underwent complete surgical resection and had been pathologically confirmed with BD-IPMN or mixed-type IPMN.

BD-IPMN was defined as a $>5$-mm-sized pancreatic cyst that communicated with the MPD, which was not dilated on radiologic examination, such as multidetector computed tomography (MDCT), MRI, or endoscopic ultrasonography (EUS) [9]. Mixed-type IPMN was defined as BD-IPMN with MPD dilatation $>5 \mathrm{~mm}$ without any obstructive lesions. Clinical valuables included the patient's age, sex, preoperative MPD size, IPMN diameter measured by radiology, IPMN type, surgery type, location of IPMN, pathologic grade, mural nodule, cyst wall thickness, lymph node enlargement, jaundice, preoperative serum CEA and CA 19-9 levels, and cyst growth rates.

This study was approved by the Institutional Review Board (approval number: C-1609-055-790), and all patients consented to participate in this study.

\section{Diagnostic modalities}

All patients who were diagnosed with suspected IPMN underwent MDCT using either the Brilliance 64 (Philips Medical Systems, Cleveland, OH, USA) or LightSpeed Ultra (GE Healthcare, Little Chalfont, UK). MRI (Magnetom Verio; Siemens Healthcare, Erlangen, Germany) or EUS was performed as required. The MPD and cyst diameter were measured at the maximal point, on the 3-mm-thick axial and coronal images of the portal venous phase, using a picture archiving and communication system (Marosis M-view; Marotech, Seoul, Korea). Each radiologic parameter was reviewed by one surgeon and one radiologist.

\section{Pathologic evaluation}

Specimens were serially transected at a thickness of $5-7 \mathrm{~mm}$ and all slides were reviewed by a pancreatic pathology specialist in our institute. The grade of IPMN was classified by 2015 WHO criteria [13]. Low-grade dysplasia was considered as benign, and high-grade dysplasia (HGD) and IPMN with an associated invasive carcinoma were considered as malignant.

\section{Statistical analysis}

Categorical variables were compared using the chi-square test. Continuous variables were compared using Student t-test. Variables for which $\mathrm{P}<0.05$ in univariate analysis were entered into a multivariate logistic regression (LR) model to find significant predictors and estimate the hazard ratios (HRs) for the corresponding predictors. Data was considered statistically significant when $\mathrm{P}<0.05$ in 2-tailed testing.

The diagnostic performance of the 2012 and 2017 ICG were compared based on the predictability of malignancy, which was measured as the area under the receiver operating curves (AUCs). Receiver operating curves were calculated using tenfold crossvalidation of each of 3 statistical models: LR, support vector machine (SVM), and random forest (RF). As the numbers of patients with benign and malignant tumors were different, the same number of individuals from the benign group as in the malignant group were randomly selected during each tenfold cross-validation. This procedure was repeated 100 times and mean AUCs were calculated for both guidelines. A tenfold crossvalidation using the LR model was performed to determine statistically driven cutoff values for continuous predictors.

All statistical analyses used IBM SPSS Statistics ver. 22.0 (IBM Co., Armonk, NY, USA) and R ver. 3.3.3 (R Foundation for Statistical Computing, Vienna, Austria). 


\section{RESULTS}

A total of 448 patients were included in this study (Table 1). Mean age was 64.7 years and the male to female ratio was 1.4. The majority of lesions were located on the pancreatic head (55.6\%), with a mean cyst size of $32.0 \mathrm{~mm}$, and a mean MPD diameter of $5 \mathrm{~mm}$.

Out of 448 patients, 148 patients (33.0\%) had a pathologically malignant lesion (HGD $[\mathrm{n}=58,12.9 \%$ or IPMN with invasive carcinoma [n $=90,20.1 \%]$ ). Compared to patients with benign lesions, their age was typically older (66.3 years vs. 63.9 years, $P$ $=0.008)$, their cyst sizes were larger $(35.0 \mathrm{~mm}$ vs. $30.5 \mathrm{~mm}, \mathrm{P}=$ 0.002), and their MPD diameters were larger $(7 \mathrm{~mm}$ vs. $4 \mathrm{~mm}$, $\mathrm{P}=0.001)$. Malignant lesions were more frequently located on the pancreatic head versus the benign lesions $(62.8 \%$ vs. $52.0 \%$, $\mathrm{P}=0.001)$.

\section{Diagnostic parameters predicting malignant $\mathrm{BD} /$ mixed type IPMN}

Table 2 shows the univariate analysis and the diagnostic values of each parameter. Parameters significantly associated with the malignancy in the univariate analysis included MPD dilatation $(>5 \mathrm{~mm})(54.7 \%$ vs. $22.0 \%, \mathrm{P}=0.001)$, the presence of mural nodule $(62.8 \%$ vs. $12.7 \%, P=0.001)$, thickened cystic wall (38.5\% vs. $10.3 \%, \mathrm{P}=0.001)$, abrupt change in MPD diameter ( $19.3 \%$ vs. $4.1 \%, \mathrm{P}=0.001)$, lymphadenopathy $(5.2 \%$ vs. $0.4 \%, \mathrm{P}=$ 0.002), elevated CEA level ( $>5 \mathrm{ng} / \mathrm{mL})(6.8 \%$ vs. $2.3 \%, \mathrm{P}=0.046)$, and elevated CA $19-9$ level (>37 unit $/ \mathrm{mL})(60.9 \%$ vs. $29.7 \%, \mathrm{P}=$ 0.012). For the 87 patients who underwent imaging surveillance for at least 2 years, lesions showing $>5 \mathrm{~mm}$ growth over 2 years were more frequently malignant compared to benign lesions (60.9\% vs. $29.7 \%, \mathrm{P}=0.012)$. The presence of a mural nodule had the highest balanced accuracy (79.2\%), followed by elevated CA 19-9 level (72.9\%) and thickened cystic wall (72.7\%). A cystic growth rate $>5 \mathrm{~mm}$ over 2 years had a balanced accuracy of $67.8 \%$.

In our multivariate analysis, the presence of mural nodule
(HR, 9.12; 95\% confidence interval [CI], 4.60-18.09; $\mathrm{P}=0.001$ ), MPD dilatation ( $>5 \mathrm{~mm}$ ) (HR, 5.32; $95 \% \mathrm{CI}, 2.67-10.60 ; \mathrm{P}=$ 0.001 ), thickened cystic wall (HR, 3.40; 95\% CI, 1.51-7.63; P = 0.003), and elevated CA 19-9 level (>37 unit/mL) (HR, 5.25; $95 \%$ $\mathrm{CI}, 2.05-13.42$; $\mathrm{P}=0.001$ ) were independent risk factors for malignant branch-duct (BD)/mixed type IPMN (Table 3). Cyst growth rate was removed from the LR model because only 87 patients (19.4\%) were evaluated.

After the risk factors for predicting malignant lesion were selected, diagnostic values were calculated according to the number of risk factors (Table 4). Sensitivity, specificity, positive predictive value (PPV), and balanced accuracy increased as the number of risk factors increased.

\section{Comparison of the diagnostic performance of the 2012 and 2017 ICG}

AUCs using three statistical methods (LR, SVM, and RF) were evaluated based on the 87 patients who had data on cystic growth rates. All of the AUCs were higher with the 2017 ICG than with the 2012 ICG (SVM, 0.686 vs. 0.650; LR, 0.784 vs. 0.746; RF, 0.787 vs. 0.758$)$.

\section{DISCUSSION}

Following the 2006 and 2012 ICG for the management of IPMN [2,3], research intensified to gather the evidence needed to support these guidelines. Particularly in the case of $\mathrm{BD} / \mathrm{mixed}$ type IPMN, where large-scaled retrospective studies suggest that the BD/mixed type IPMN has only a 5\%-20\% likelihood of invasiveness [14-19], the question of whether surgical resection or regular surveillance is preferable is an important issue. $\mathrm{BD} /$ mixed type IPMN without high-risk or worrisome features has been defined by the 2012 guidelines not to warrant surgical resection [3]. The associated decline in unnecessary surgeries had led to a decrease in surgery-related mortality, morbidity, and medical costs.

In 2015, the AGA released new guidelines with lesser

Table 1. Baseline characteristics of patients with branch-duct/mixed type intraductal papi llary mucinous neoplasm

\begin{tabular}{|c|c|c|c|c|}
\hline Characteristic & Total $(n=448)$ & Benign IPMN ( $\mathrm{n}=300)$ & Malignant IPMN $(\mathrm{n}=148)$ & P-value \\
\hline Age (yr) & $64.7 \pm 8.8$ & $63.9 \pm 8.5$ & $66.3 \pm 9.3$ & 0.008 \\
\hline Sex, male:female & $263: 185$ & $178: 122$ & $85: 63$ & 0.760 \\
\hline Follow-up period (mo) & $124 \pm 71$ & $124 \pm 61.0$ & $125 \pm 95$ & 0.901 \\
\hline Location (\%) & & & & 0.001 \\
\hline Head & $249(55.6)$ & $156(52.0)$ & $93(62.8)$ & \\
\hline Body or tail & $167(37.3)$ & $128(42.7)$ & $39(26.4)$ & \\
\hline Diffuse & $32(7.1)$ & $16(5.3)$ & $16(10.8)$ & \\
\hline Cyst size (mm) & $32.0 \pm 14.0$ & $30.5 \pm 13.0$ & $35.0 \pm 15.4$ & 0.002 \\
\hline MPD diameter (mm) & $0.5 \pm 0.4$ & $0.4 \pm 0.3$ & $0.7 \pm 0.5$ & 0.001 \\
\hline
\end{tabular}

Values are presented as mean \pm standard deviation or number $(\%)$.

IPMN, intraductal papillary mucinous neoplasm; MPD, main pancreatic duct. 
aggressive resection criteria and more conservative surveillance criteria than those of the 2012 guidelines [5]. However, these changes were not without controversy. In terms of detecting malignant IPMN, one study reported that $45 \%$ of malignant IPMNs were not detected following the AGA guidelines [20]. In regards to the surveillance, new-onset pancreatic cancer [21], and the IPMNs with new-onset high risk stigmata and/or worrisome features were observed beyond 5 years of surveillance [7]. One study recommended continued surveillance beyond 5 years because the overall risk rate of malignancy was $8 \%$ during 10 years of surveillance [6]. The present guideline revision was focused on addressing these issues [9].

In 2017, the ICG for IPMN were revised to include 2 new factors: elevated serum CA 19-9 level and cyst growth rate

Table 2. Univariate analysis and diagnostic value of each parameter

\begin{tabular}{|c|c|c|c|c|c|c|c|c|}
\hline Variable & $\begin{array}{c}\text { Benign } \\
\text { IPMN, n (\%) }\end{array}$ & $\begin{array}{c}\text { Malignant } \\
\text { IPMN, n (\%) }\end{array}$ & P-value & $\begin{array}{l}\text { Sensitivity } \\
(\%)\end{array}$ & $\begin{array}{l}\text { Specificity } \\
(\%)\end{array}$ & $\begin{array}{l}\text { PPV } \\
(\%)\end{array}$ & $\begin{array}{l}\text { NPV } \\
(\%)\end{array}$ & $\begin{array}{c}\text { Accuracy } \\
(\%)\end{array}$ \\
\hline \multicolumn{9}{|l|}{ Cyst size, $\geq 3 \mathrm{~cm}$} \\
\hline Yes & $139(46.3)$ & $83(56.1)$ & 0.057 & 56.1 & 53.7 & 37.4 & 71.2 & 54.5 \\
\hline No & $161(53.7)$ & $65(43.9)$ & & & & & & \\
\hline \multicolumn{9}{|c|}{ MPD diameter, $>5 \mathrm{~mm}$} \\
\hline Yes & $66(22.0)$ & $81(54.7)$ & 0.001 & 54.7 & 78.0 & 55.1 & 77.7 & 70.3 \\
\hline No & $234(78.0)$ & $67(45.3)$ & & & & & & \\
\hline \multicolumn{9}{|l|}{ Mural nodule } \\
\hline Yes & $38(12.7)$ & $93(62.8)$ & 0.001 & 62.8 & 87.3 & 71.0 & 82.7 & 79.2 \\
\hline No & $262(87.3)$ & $55(37.2)$ & & & & & & \\
\hline \multicolumn{9}{|l|}{ Thickened cystic wall } \\
\hline Yes & $28(10.3)$ & $52(38.5)$ & 0.001 & 38.5 & 89.7 & 65.0 & 74.5 & 72.7 \\
\hline No & $243(89.7)$ & $83(61.5)$ & & & & & & \\
\hline \multicolumn{9}{|c|}{$\begin{array}{l}\text { Abrupt change in MPD } \\
\text { diameter }\end{array}$} \\
\hline Yes & $11(4.1)$ & $26(19.3)$ & 0.001 & 19.3 & 95.9 & 70.3 & 70.5 & 70.4 \\
\hline No & $260(95.9)$ & $109(80.7)$ & & & & & & \\
\hline \multicolumn{9}{|l|}{ Lymphadenopathy } \\
\hline Yes & $1(0.4)$ & $7(5.2)$ & 0.002 & 5.2 & 99.6 & 87.5 & 67.8 & 68.2 \\
\hline No & $270(99.6)$ & $128(94.8)$ & & & & & & \\
\hline \multicolumn{9}{|l|}{$\mathrm{CEA},>5 \mathrm{ng} / \mathrm{mL}$} \\
\hline Yes & $6(2.3)$ & $9(6.8)$ & 0.046 & 6.8 & 97.7 & 60.0 & 67.6 & 67.3 \\
\hline No & $256(97.7)$ & $123(93.2)$ & & & & & & \\
\hline \multicolumn{9}{|l|}{ CA $19-9,>37 \mathrm{U} / \mathrm{mL}$} \\
\hline Yes & $22(7.7)$ & $51(34.9)$ & 0.001 & 34.9 & 92.3 & 69.9 & 73.5 & 72.9 \\
\hline No & $263(92.3)$ & $95(65.1)$ & & & & & & \\
\hline \multicolumn{9}{|c|}{ Cyst growth rate, $>5 \mathrm{~mm} / 2 \mathrm{yr}^{\mathrm{a})}$} \\
\hline Yes & $19(29.7)$ & $14(60.9)$ & 0.012 & 60.9 & 70.3 & 42.4 & 83.3 & 67.8 \\
\hline No & $45(70.3)$ & $9(39.1)$ & & & & & & \\
\hline
\end{tabular}

PPV, positive predictive value; NPV, negative predictive value; MPD, main pancreatic duct.

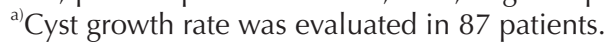

Table 3. Predictive factors for malignant branch-duct type intraductal papillary mucinous neoplasm

\begin{tabular}{llll}
\hline \multicolumn{1}{c}{ Variable } & HR & \multicolumn{1}{c}{$95 \% \mathrm{Cl}$} & P-value \\
\hline Mural nodule & 9.12 & $4.60-18.09$ & 0.001 \\
MPD diameter, $>5 \mathrm{~mm}$ & 5.32 & $2.67-10.60$ & 0.001 \\
Thickened cyst wall & 3.40 & $1.51-7.63$ & 0.003 \\
CA 19-9, $>37 \mathrm{U} / \mathrm{mL}$ & 5.25 & $2.05-13.42$ & 0.001 \\
Abrupt change in MPD diameter & 2.45 & $0.78-7.94$ & 0.124 \\
Lymphadenopathy & 3.79 & $0.31-46.74$ & 0.298 \\
CEA, $>5 \mathrm{ng} / \mathrm{mL}$ & 2.90 & $0.80-10.45$ & 0.104
\end{tabular}

$\mathrm{HR}$, hazard ratio; $\mathrm{Cl}$, confidence interval; MPD, main pancreatic duct. 
Table 4. Diagnostic value according to the number of risk factors

\begin{tabular}{|c|c|c|c|c|c|c|c|c|}
\hline $\begin{array}{l}\text { No. of } \\
\text { factors }\end{array}$ & $\begin{array}{c}\text { Benign } \\
\text { IPMN, n (\%) }\end{array}$ & $\begin{array}{l}\text { Malignant } \\
\text { IPMN, n (\%) }\end{array}$ & P-value & $\begin{array}{l}\text { Sensitivity } \\
(\%)\end{array}$ & $\begin{array}{l}\text { Specificity } \\
(\%)\end{array}$ & $\begin{array}{l}\text { PPV } \\
(\%)\end{array}$ & $\begin{array}{l}\text { NPV } \\
(\%)\end{array}$ & $\begin{array}{c}\text { Balanced } \\
\text { accuracy (\%) }\end{array}$ \\
\hline 0 & $70(35.0)$ & $2(1.8)$ & - & - & - & - & - & - \\
\hline 1 & $81(40.5)$ & $21(18.8)$ & 0.001 & 91.3 & 46.4 & 20.6 & 97.2 & 68.0 \\
\hline 2 & 37 (18.5) & $25(22.3)$ & $<0.001$ & 92.6 & 65.4 & 40.3 & 97.2 & 79.0 \\
\hline 3 & $8(4.0)$ & $28(25.0)$ & $<0.001$ & 93.3 & 89.7 & 77.8 & 97.2 & 91.5 \\
\hline$\geq 4$ & $3(1.5)$ & $23(20.5)$ & $<0.001$ & 94.7 & 94.6 & 90.0 & 97.2 & 94.7 \\
\hline
\end{tabular}

IPMN, intraductal papillary mucinous neoplasm; PPV, positive predictive value; NPV, negative predictive value.

[9]. Although the role of elevated CEA has been controversial, elevated serum CA 19-9 has been reported as a useful predictive marker for malignant IPMNs [12,15,22]. In the present study, elevated serum CA 19-9 (>37 U/mL) was strongly associated with malignancy in a multivariate analysis (HR, 5.25; 95\% CI, 2.05-13.42; $\mathrm{P}<0.001$ ) (Table 3), and had a high diagnostic accuracy $(72.9 \%$, Table 2). Laboratory criteria benefit greatly from their relative objectivity compared to preoperative radiologic interpretation. Therefore, the introduction of serum CA 19-9 to the guidelines would be reasonable and improve diagnostic accuracy.

Some studies suggested that a cyst growth rate $>2-4 \mathrm{~mm}$ per year could be a risk factor for malignant $\mathrm{BD} /$ mixed type IPMN $[10,11,15]$. One large-scale study revealed that benign BD-IPMNs observed for $>5$ years grew slowly at a rate of only $0.8 \mathrm{~mm}$ per year [23]. Therefore, growth of $2-4 \mathrm{~mm}$ per year is relatively rapid. In the 2017 ICG, the cyst growth rate $>5 \mathrm{~mm}$ in 2 years was added to the worrisome features. In the present study, the cyst growth rate $>5 \mathrm{~mm}$ in 2 years was observed more frequently in malignant lesions, and the predictive accuracy was $67.8 \%$ in the univariate analysis (Table 2). However, it was not entered into the multivariate analysis due to insufficient data. In order to overcome this limitation, large-cohort studies with long-term surveillance data should be performed.

In our multivariate analysis, mural nodule, dilated MPD, thickened cyst wall, and elevated CA 19-9 level $(>37 \mathrm{U} / \mathrm{mL})$ were associated with malignancy (Table 3). The diagnostic validity was evaluated according to the number of risk factors (Table 4). According to these results, if a patient had at least 2 risk factors, the resection would be appropriate because the PPV was over $40 \%$ and the balanced accuracy was nearly $80 \%$. Furthermore, the possibility of malignant IPMN was greater than $90 \%$ when at least three risk factors were present. Several nomograms able to quantify the possibility of malignancy were released, which combined the risk factors based on the multivariate LR [24,25]. Therefore, these nomograms can be valuable tools to accurately predict malignant IPMNs.

Whether cystic size is a risk factor or not remains controversial. In the 2017 ICG, a cyst size $>3 \mathrm{~cm}$ is a worrisome feature [9]. However, recent studies argue that cystic size is not associated with malignant BD-IPMNs [26-28]. In the present study, cystic size was not associated with malignancy in the univariate analysis, and it had the lowest diagnostic accuracy (54.5\%) among the potential risk factors (Table 2). Cyst size alone could not be an absolute indication for resection [4]. However, larger IPMNs tended to have more risk factors and, thus, greater malignant potential [23]. Therefore, larger IPMNs should be observed carefully with standardized protocols.

Jang et al. [4] reported the superior diagnostic performance of the 2012 ICG over the 2006 ICG by using 3 statistical methods (LR, SVM, and RF). In the present study, the same methods were utilized and the AUC values of the 2017 ICG were further improved over the 2012 ICG. The introduction of elevated serum CA 19-9 and a rapid cyst growth rate increased the diagnostic performance of the 2017 ICG.

Despite the strengths of this study, it does have its limitations. It was a single-center retrospective study. All patients had pathologic diagnosis after surgical resection. Many small cysts without worrisome features were excluded due to the lack of a pathologic diagnosis. Only patients who had both postoperative radiologic surveillance data and pathologic results were included in the analysis of cystic growth rate. Therefore, the number of patients available for evaluating cyst growth rate was limited; and therefore, the LR could not include this feature. A future study based on long-term regular surveillance would be needed to more fully address the role of cystic growth rate. Despite of these shortcomings, all the patients in the present study underwent diagnostic examination with standardized protocols, and the radiologic parameters were reviewed again by both a surgeon and a radiologist to improve diagnostic concordance.

In conclusion, the new 2017 ICG for IPMN are clinically valid with improved diagnostic performance over the previous 2012 guidelines. The introduction of elevated serum CA 19-9 level and cyst growth rate to the 2017 guidelines is appropriate.

\section{CONFLICTS OF INTEREST}

No potential conflict of interest relevant to this article was reported. 


\section{ACKNOWLEDGEMENTS}

This research was supported by grants of the Korea Health Technology R\&D Project through the Korea Health Industry Development Institute (KHIDI), funded by the Ministry of Health \& Welfare, Republic of Korea (grant number: HI16C2037), and by the Collaborative Genome Program for Fostering New Post-Genome Industry of the National Research Foundation funded by the Ministry of Science and ICT (NRF2017M3C9A5031591).

\section{REFERENCES}

1. Kloppel G, Solcia E, Longnecker D, Capella C, Sobin L. Histological typing of tumors of the exocrine pancreas, In: Kloppel G, Solcia E, Longnecker D, Capella C, Sobin L. Histological classification of tumors of the exocrine pancreas. 2nd ed. Berlin: Springer-Verlag; 1996. p. 7-9.

2. Tanaka M, Chari S, Adsay V, Fernandezdel Castillo C, Falconi M, Shimizu M, et al. International consensus guidelines for management of intraductal papillary mucinous neoplasms and mucinous cystic neoplasms of the pancreas. Pancreatology 2006;6:17-32.

3. Tanaka M, Fernandez-del Castillo C. Adsay V, Chari S, Falconi M, Jang JY, et al. International consensus guidelines 2012 for the management of IPMN and MCN of the pancreas. Pancreatology 2012;12:18397.

4. Jang JY, Park T, Lee S, Kang MJ, Lee SY, Lee KB, et al. Validation of international consensus guidelines for the resection of branch duct-type intraductal papillary mucinous neoplasms. Br J Surg 2014;101:686-92.

5. Vege SS, Ziring B, Jain R, Moayyedi P; Clinical Guidelines Committee; American Gastroenterology Association. American gastroenterological association institute guideline on the diagnosis and management of asymptomatic neoplastic pancreatic cysts. Gastroenterology 2015;148: 819-22.

6. Pergolini I, Sahora K, Ferrone CR, MoralesOyarvide V, Wolpin BM, Mucci LA, et al. Long-term risk of pancreatic malignancy in patients with branch duct intraductal papillary mucinous neoplasm in a referral center. Gastroenterology 2017;153:1284 94.e1.

7. Crippa S, Pezzilli R, Bissolati M, Capurso G, Romano L, Brunori MP, et al. Active surveillance beyond 5 years is required for presumed branch-duct intraductal papillary mucinous neoplasms undergoing non-operative management. Am J Gastroenterol 2017;112:1153-61.

8. Del Chiaro M, Ateeb Z, Hansson MR, Rangelova E, Segersvard R, Kartalis N, et al. Survival analysis and risk for progression of intraductal papillary mucinous neoplasia of the pancreas (IPMN) under surveillance: a single-institution experience. Ann Surg Oncol 2017;24:1120-6.

9. Tanaka M, Fernandez-Del Castillo C, Kamisawa T, Jang JY, Levy P, Ohtsuka T, et al. Revisions of international consensus Fukuoka guidelines for the management of IPMN of the pancreas. Pancreatology 2017;17:738-53.

10. Kang MJ, Jang JY, Kim SJ, Lee KB, Ryu JK, Kim YT, et al. Cyst growth rate predicts malignancy in patients with branch duct intraductal papillary mucinous neoplasms. Clin Gastroenterol Hepatol 2011;9:87-93.

11. Kwong WT, Lawson RD, Hunt G, Fehmi SM, Proudfoot JA, Xu R, et al. Rapid growth rates of suspected pancreatic cyst branch duct intraductal papillary mucinous neoplasms predict malignancy. Dig Dis Sci 2015;60:2800-6.

12. Kim JR, Jang JY, Kang MJ, Park T, Lee SY, Jung W, et al. Clinical implication of serum carcinoembryonic antigen and carbohydrate antigen 19-9 for the prediction of malignancy in intraductal papillary mucinous neoplasm of pancreas. J Hepatobiliary Pancreat Sci 2015;22:699-
707.

13. Basturk O, Hong SM, Wood LD, Adsay NV, Albores-Saavedra J, Biankin AV, et al. A revised classification system and recommendations from the baltimore consensus meeting for neoplastic precursor lesions in the pancreas. Am J Surg Pathol 2015;39:1730-41.

14. Schmidt CM, White PB, Waters JA, Yiannoutsos CT, Cummings OW, Baker $\mathrm{M}$, et al. Intraductal papillary mucinous neoplasms: predictors of malignant and invasive pathology. Ann Surg 2007;246:644-51.

15. Hwang DW, Jang JY, Lee SE, Lim CS, Lee KU, Kim SW. Clinicopathologic analysis of surgically proven intraductal papillary mucinous neoplasms of the pancreas in SNUH: a 15-year experience at a single academic institution. Langenbecks Arch Surg 2012;397:93-102.

16. Crippa S, Fernandez-Del Castillo C, Salvia R, Finkelstein D, Bassi C, Dominguez I, et al. Mucin-producing neoplasms of the pancreas: an analysis of distinguishing clinical and epidemiologic characteristics. Clin Gastroenterol Hepatol 2010;8:213-9.

17. Rodriguez JR, Salvia R, Crippa S, Warshaw AL, Bassi C, Falconi M, et al. Branch-duct intraductal papillary mucinous neoplasms: observations in 145 patients who underwent resection. Gastroenterology 2007;133:72-9.

18. Jang JY, Kim SW, Lee SE, Yang SH, Lee KU, Lee YJ, et al. Treatment guidelines for branch duct type intraductal papillary mucinous neoplasms of the pancreas: when can we operate or observe? Ann Surg Oncol 2008;15:199-205.

19. Kanno A, Satoh K, Hirota M, Hamada S, 
Umino J, Itoh H, et al. Prediction of invasive carcinoma in branch type intraductal papillary mucinous neoplasms of the pancreas. J Gastroenterol 2010;45:952-9.

20. Singhi AD, Zeh HJ, Brand RE, Nikiforova MN, Chennat JS, Fasanella KE, et al. American Gastroenterological Association guidelines are inaccurate in detecting pancreatic cysts with advanced neoplasia: a clinicopathologic study of 225 patients with supporting molecular data. Gastrointest Endosc 2016;83:1107-17.e2.

21. Imbe K, Nagata N, Hisada Y, Takasaki Y, Sekine K, Mishima S, et al. Validation of the American Gastroenterological Association guidelines on management of intraductal papillary mucinous neoplasms: more than 5 years of followup. Eur Radiol 2018;28:170-8.

22. Fritz S, Hackert T, Hinz U, Hartwig W, Buchler MW, Werner J. Role of serum carbohydrate antigen 19-9 and carcinoembryonic antigen in distinguishing between benign and invasive intraductal papillary mucinous neoplasm of the pancreas. Br J Surg 2011;98:104-10.

23. Han Y, Lee H, Kang JS, Kim JR, Kim HS, Lee JM, et al. Progression of pancreatic branch duct intraductal papillary mucinous neoplasm associates with cyst size. Gastroenterology 2018;154:576-84.

24. Jang JY, Park T, Lee S, Kim Y, Lee SY, Kim SW, et al. Proposed nomogram predicting the individual risk of malignancy in the patients with branch duct type intraductal papillary mucinous neoplasms of the pancreas. Ann Surg 2017;266:1062-8.

25. Attiyeh MA, Fernandez-Del Castillo C, Al Efishat M, Eaton AA, Gonen M, Batts R, et al. Development and validation of a multiinstitutional preoperative nomogram for predicting grade of dysplasia in intraductal papillary mucinous neoplasms (IPMNs) of the pancreas: a report from the pancre- atic surgery consortium. Ann Surg 2018; 267:157-63.

26. Kim KW, Park SH, Pyo J, Yoon SH, Byun $\mathrm{JH}$, Lee MG, et al. Imaging features to distinguish malignant and benign branch-duct type intraductal papillary mucinous neoplasms of the pancreas: a meta-analysis. Ann Surg 2014:259:72-81.

27. Ridtitid W, DeWitt JM, Schmidt CM, Roch A, Stuart JS, Sherman S, et al. Management of branch-duct intraductal papillary mucinous neoplasms: a large single-center study to assess predictors of malignancy and long-term outcomes. Gastrointest Endosc 2016;84:436-45.

28. Yamada S, Fujii T, Murotani K, Kanda M, Sugimoto H, Nakayama G, et al. Comparison of the international consensus guidelines for predicting malignancy in intraductal papillary mucinous neoplasms. Surgery 2016;159:878-84. 